\title{
Comparative chemometric analysis of fluorescence and near infrared spectroscopies for authenticity confirmation and geographical origin of Argentinean extra virgin olive oils
}

\author{
Ana M. Jiménez-Carvelo ${ }^{\mathrm{a}}$, Valeria A. Lozano ${ }^{\mathrm{b}, *}$, Alejandro C. Olivieri ${ }^{\mathrm{b}}$ \\ a Department of Analytical Chemistry, University of Granada, c/Fuentenueva, s.n, E-18071, Granada, Spain \\ ${ }^{\mathbf{b}}$ Instituto de Química Rosario (IQUIR-CONICET), Facultad de Ciencias Bioquímicas y Farmacéuticas, Universidad Nacional de Rosario, Suipacha 531, S2002LRK, \\ Rosario, Argentina
}

\section{A R T I C L E I N F O}

\section{Keywords:}

NIR spectroscopy

Fluorescence

Extra virgin olive oil

Adulteration

Geographical origin

Chemometrics

\begin{abstract}
A B S T R A C T
Extra virgin olive oil (EVOO) is widely used in gastronomy because of its healthy properties, and is a candidate to be adulterated with other vegetable oils to reduce costs. This work shows the results of applying near infrared (NIR) and fluorescence excitation-emission matrix spectroscopies, coupled to chemometric tools, to authenticate and validate the geographic origin of Argentinean EVOO samples. For each spectral data set, principal component analysis (PCA) was applied to both first-order NIR and second-order fluorescence data, partial least squares-discriminant analysis (PLS1-DA) to NIR data, and the multidimensional version of the latter (NPLS-DA) to fluorescence data. The results of the study of sixty EVOO samples of known and unknown registered designation of origin (RDO), as well as artificial samples adulterated with other edible oils, showed that: (1) fluorescence spectroscopy was unable to determine the RDO of all EVOO samples, in contrast to NIR (100\% classified correctly), and (2) fluorescence data provide only slightly better results than NIR spectroscopy to detect EVOO adulterations with other vegetable edible oils.
\end{abstract}

\section{Introduction}

Extra virgin olive oil (EVOO) is obtained from the fruit of the olive tree (Olea europaea L.), and is recognized for its functional properties, due to the high content of monounsaturated fatty acids and natural antioxidants, such as vitamins A and E. Argentina is the tenth world producer of olive oil, occupying the first place in the American continent. According to the International Olive Council (IOC) (IOC, 2011), the estimated area of olive plantations is around 105,000 hectares and the main producing provinces are Catamarca, La Rioja, Mendoza, San Juan, Córdoba and Buenos Aires. About $60 \%$ of the production is destined to the production of olive oil, especially of the extra virgin quality, which represents almost $1 \%$ of the total worldwide production.

The Argentinean Food Code (CAA) (Argentinean Food Code (CAA) chapter VII, 2017) establishes that an olive oil is considered to be the one corresponding exactly to its designation and, consequently, the presence of any other oils are not allowed. Also, the identification of the olive oil origin (country and province) must be included in the product label as mandatory information (Argentinean Food Code (CAA) chapter $\mathrm{V}$, 2017). For this reason, it is important to confirm its provenance, because if not fulfilled, it would constitute a false authentication of the product. Additionally, a current problem in the olive industry is the adulteration of EVOO (the best quality olive oil) with lower price/ quality oils, such as seed oils (sunflower, corn, and soybean) (Firestone, 2001). The most widely used analytical methods to detect EVOO adulteration with refined oil are gas chromatography (GC) (Cercaci, Rodriguez-Estrada, \& Lercker, 2003; Flores, Ruiz Del Castillo, Blanch, \& Herraiz, 2006) and high performance liquid chromatography (HPLC) (Fasciotti \& Pereira Netto, 2010; Jabeur et al., 2014). These methods require rigorous extraction steps, the use of significant amounts of organic solvents and long analysis time. Fluorescence spectroscopy techniques have been addressed to the search for adulteration, varieties and chemical compounds (vitamins, chlorophylls and other important compounds) of EVOO (Zandomeneghi, Carbonaro, \& Caffarata, 2005), frequently associated with chemometric analysis (Durán Merás, Domínguez Manzano, Airado Rodríguez, \& Muñoz de la Peña, 2018; Guimet, Ferré, Boqué, \& Rius, 2004; Poulli, Mousdis, \& Georgiou, 2005). Near infrared (NIR) spectroscopy supported by chemometric approaches is a rapid, simple, inexpensive and non-destructive methodology to detect fraudulent practices. This method has been employed

\footnotetext{
* Corresponding author.

E-mail address: lozano@iquir-conicet.gov.ar (V.A. Lozano).
} 
to study the composition, authenticity and to verify quality-levels such as the registered designation of origin (RDO) (Galtier et al., 2007; Nunes, 2014; Woodcock, Downey, \& O'Donnell, 2008). NIR is also regarded as attractive in research, control and industrial laboratories, because of its greenness (Gałuszka, Migaszewski, \& Namieśnik, 2013; Moros, Garrigues, \& Guardia, 2010). It may allow one to develop untargeted fingerprint methods for the classification of samples based on spectral patterns, without identification or quantitation of specific components. Spectroscopic techniques show promising potential for sample classification even with a limited number of samples (Ellis et al., 2012).

The consumer interest in EVOO as a source of healthy food has increased in recent years. Due to its nutritional value and its economic importance, it is essential to have simple and green methodologies to identify possible adulterations, authenticity and RDO. In this context, the aim of the present work is to compare two fingerprint methods, excitation-emission fluorescence matrix (EEFM) and NIR spectroscopies, which might be useful for validation of quality-levels of EVOO samples. The results obtained by means of different chemometric models, such as principal component analysis (PCA) and partial least squares-discriminant analysis (PLS-DA), are discussed in detail.

\section{Experimental}

\subsection{Instrumentation}

\subsubsection{NIR spectra}

NIR spectra were collected in duplicate at room temperature in the transflectance mode with a $0.5 \mathrm{~mm}$ gold reflector and slurry cup quartz for liquids, using a FOSS NIRS DS2500 spectrometer (FOSS, Hillerød, Denmark) equipped with a monochromator and a dual Si and PbS detector. The spectra data were recorded with the wavelength mode, ranging from 1100 to $2500 \mathrm{~nm}$ with a spectral resolution of $0.5 \mathrm{~nm}$.

\subsubsection{Fluorescence excitation-emission matrices}

Fluorescence measurements were performed on a Perkin Elmer LS 55 luminescence spectrometer, equipped with a xenon discharge lamp (equivalent to $20 \mathrm{~kW}$ for $8 \mu$ duration) and connected to a PC microcomputer. Excitation and emission slit widths were of $5 \mathrm{~nm}$ using $1.00 \mathrm{~cm}$ quartz cells. The photomultiplier tube sensitivity was fixed at $800 \mathrm{~V}$ and the scan rate at $1500 \mathrm{~nm} \mathrm{~min}^{-1}$. The EEFMs were measured in duplicate in the ranges $300-410 \mathrm{~nm}$ (each $5 \mathrm{~nm}$ ) for excitation, and $420-700 \mathrm{~nm}$ (each $0.5 \mathrm{~nm}$ ) for emission, yielding matrices of size $23 \times 561$ data points. Data were saved in ASCII format and transferred to a PC for subsequent chemometric analysis.

\subsection{Samples}

A total of 60 EVOO samples were obtained from supermarkets, fairs and shops from different Argentinean cities. 52 EVOO samples came from six Argentinean RDO and 8 EVOO samples have unknown RDO. Table 1 shows the RDO declared in the label of each EVOO sample. All samples were stored in amber glass bottles in the dark at room temperature until they were analyzed. The samples were analyzed in crude form, without any pretreatment or dilution.

Three additional samples of seed oils (sunflower, corn and soybean) were analyzed in crude form, and mixtures of EVOO were prepared by adding each of the three edible oils in different proportions $(30 \%, 50 \%$ and 70\%). After addition of the edible oils, the blends were manually shaken for about $30 \mathrm{~s}$ and stored until the analysis. Table 2 shows the seed oils samples and the composition of the blend oil samples.
Table 1

RDO declared in the labels of the EVOO samples.

\begin{tabular}{|c|c|c|c|c|}
\hline \multicolumn{4}{|c|}{ Sample number ${ }^{\mathrm{a}}$} & \multirow{2}{*}{$\begin{array}{l}\text { RDO } \\
\text { La Rioja }\end{array}$} \\
\hline $1-2$ & $7-8$ & $13-14$ & $19-20$ & \\
\hline $3-4$ & $9-10$ & $15-16$ & $21-22$ & \\
\hline $5-6$ & $11-12$ & $17-18$ & $23-24$ & \\
\hline $25-26$ & $31-32$ & $37-38$ & $43-44$ & Mendoza \\
\hline $27-28$ & $33-34$ & $39-40$ & $45-46$ & \\
\hline $29-30$ & $35-36$ & $41-42$ & & \\
\hline $47-48$ & $49-50$ & $51-52$ & $53-54$ & Catamarca \\
\hline $55-56$ & $61-62$ & $67-68$ & $73-74$ & Córdoba \\
\hline $57-58$ & $63-64$ & $69-70$ & & \\
\hline $59-60$ & $65-66$ & $71-72$ & & \\
\hline $75-76$ & $79-80$ & $83-84$ & $87-88$ & San Juan \\
\hline $77-78$ & $81-82$ & $85-86$ & $89-90$ & \\
\hline 91-92 & $95-96$ & $99-100$ & $103-104$ & Buenos Aires \\
\hline 93-94 & $97-98$ & 101-102 & & \\
\hline 105-106 & $109-110$ & $113-114$ & $117-118$ & Unknown \\
\hline 107-108 & $111-112$ & $115-116$ & $119-120$ & \\
\hline
\end{tabular}

a (original-duplicate).

Table 2

Seed oil samples and composition of adulterated oil samples.

\begin{tabular}{ll}
\hline Sample number $^{\mathrm{a}}$ & Composition \\
\hline $121-122$ & Corn oil (100\%) \\
$123-124$ & Blend of EVOO/Corn oil (70\%) \\
$125-126$ & Blend of EVOO/Corn oil (50\%) \\
$127-128$ & Blend of EVOO/Corn oil (30\%) \\
\hline $129-130$ & Sunflower oil (100\%) \\
$131-132$ & Blend of EVOO/Sunflower oil (70\%) \\
$133-134$ & Blend of EVOO/Sunflower oil (50\%) \\
$135-136$ & Blend of EVOO/Sunflower oil (30\%) \\
\hline $137-138$ & Soybean oil (100\%) \\
$139-140$ & Blend of EVOO/Soybean oil (70\%) \\
$141-142$ & Blend of EVOO/Soybean oil (50\%) \\
$143-144$ & Blend f EVOO/Soybean oil (30\%)
\end{tabular}

${ }^{\text {a }}$ (original-duplicate).

\subsection{Chemometric models and software}

PCA is usually the first step to detect sample groups from the measured data because it permits an overview of the data structure in a space defined by the principal components (PCs). The combination of the different PCs reveals relationships between the objects, aside from outlier objects. PCA was applied directly to first-order NIR data, whereas in the case of second-order EEFM data were first processed by parallel factor analysis (PARAFAC), a well-known multi-way model which uniquely decomposed the data for a group of EEFM in excitation, emission and relative component concentrations or scores. PCA was applied to the matrix of scores generated for each component and sample.

PLS regression can be adapted for classification, leading to a variant of discriminant analysis, PLS-DA, in which the predicted variables are categorical values. PLS1-DA is applied for first-order data and is calibrated one category at a time, whereas the multidimensional version NPLS-DA is useful for second-order data. Both models are implemented using a binary coding pattern with one bit per class (Galtier et al., 2007). 


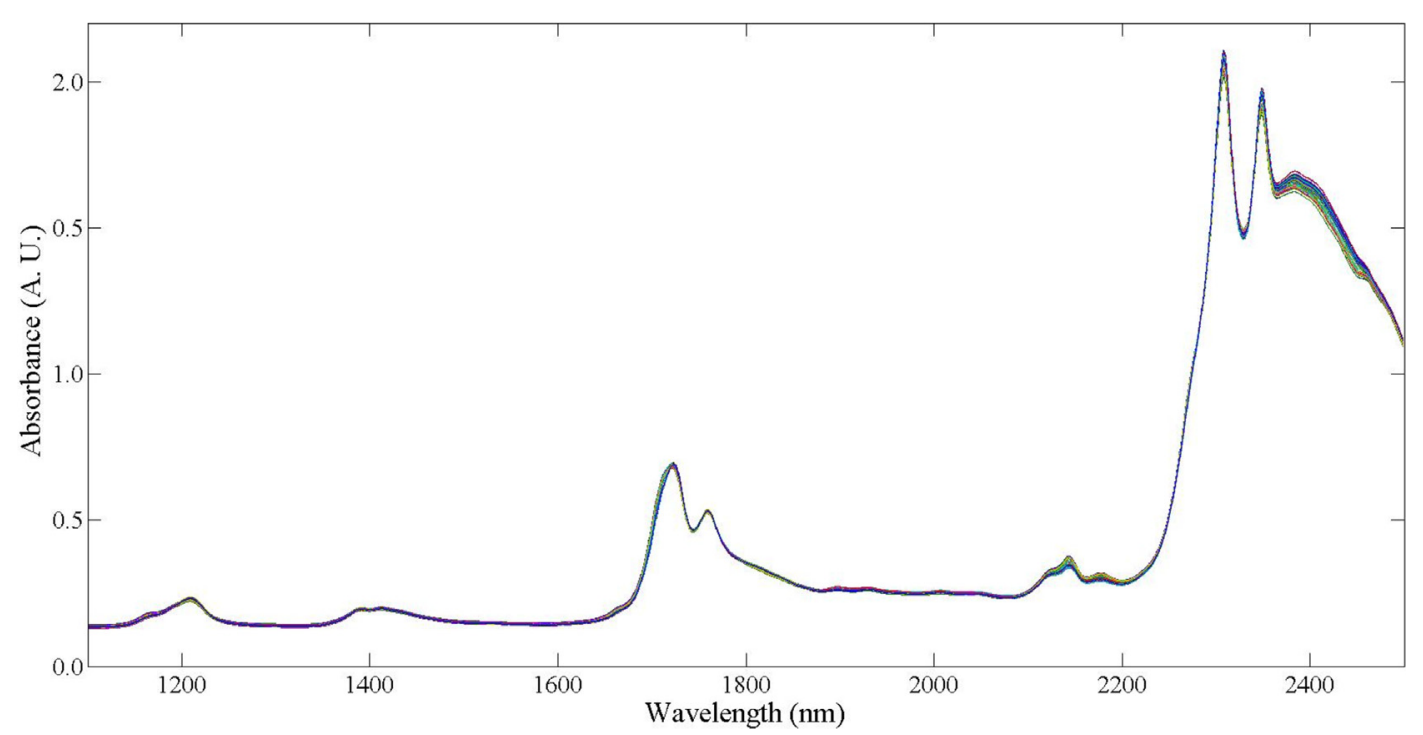

Fig. 1. NIR spectra of the 60 EVOO samples studied (by duplicate). A. U.: arbitrary units.

The theory of the applied models in the present work is well established and is detailed the relevant references: PARAFAC (Bro, 1997), PCA (Abdi \& Williams, 2010; Bro \& Smilde, 2014), PLS1-DA (Barker \& Rayens, 2003) and NPLS-DA (Arancibia, Boschetti, Olivieri, \& Escandar, 2008). PCA was run employing in-house routines written in MATLAB 7.10 (MATLAB, 2010). PARAFAC and NPLS-DA were carried out by means of the graphical interface MVC2 (Olivieri, Wu, \& Yu, 2009) and PLS1-DA was implemented using the graphical interface MVC1 (Olivieri, Goicoechea, \& Iñón, 2004), both available on the Internet (MVC1, 2018).

\section{Results and discussion}

\subsection{NIR spectroscopy}

\subsubsection{PCA}

Fig. 1 shows the 120 NIR spectra measured for the 60 EVOO samples. As can be seen, all of them are very similar, and imply a high degree of spectral correlation. The best exploratory results with PCA were obtained in the range $1100-2500 \mathrm{~nm}$, after applying first-derivative and mean centering as data pre-processing. The first two PCs explained more than $93 \%$ of the spectral variance, and the score-score plot showed some tendency to cluster in two groups (data not shown): (1) a larger one consisting of 44 EVOO samples and (2) a smaller one formed by the remaining 16 samples and located at more negative values of PC1. Most of the 16 samples constituting the smallest group were confirmed to be reported as unsafe by Argentinean Food Safety Agencies (ANMAT, 2018; ASSAI, 2018), because they do not comply with the current Food Legislation. Thus their commercialization is prohibited and it is requested not to acquire nor consume these oils in all the Argentinean territory. Therefore, we decided to include the analysis of edible oils and mixtures of them with EVOO samples (Table 2). At first sight their NIR spectra were analogous to those of authentic EVOO samples. Thus, a new PCA study was performed on the NIR spectra under the same pre-processing conditions. Fig. 2 shows the score-score plot obtained of PC1 (91.4\%) vs. PC2 (6.2\%). It was corroborated that the seed oils samples and the corresponding blends overlapped with the above mentioned 16 samples under question. The color scheme in Fig. 2 shows the EVOO samples according to their RDO (Table 1), and the seed oil and blend samples marked with a circle: red (corn), blue (sunflower) and green (soybean). Therefore, the PCA model achieved revealed some differences between the EVOO samples according to their purity, i.e. it allowed one to distinguish into adulterated oils and pure EVOO. Nevertheless, it did not separate the samples by RDO, despite the fact that, in principle, the NIR spectra carry information related to EVOO geographical origin. This may be due to the fact that there are few samples available from some regions, e.g. for Catamarca $n=4$ (see Table 1).

\subsubsection{PLS1-DA}

3.1.2.1. Classification of EVOO and adulterated olive oils. The complete data set (EVOO, edible oil samples and blends, by duplicate, $n=144$ ) was randomly split into calibration $(n=108)$ and validation $(n=36)$ sub-sets. Both replicates corresponding to the same sample were included in the same sub-set. The PLS1-DA model for classification of EVOO and adulterated olive oil samples was developed using first-derivative, standard normal variate (SNV) and mean center pre-processing, in the spectral range $1100-2200 \mathrm{~nm}$. Leave-one-out cross-validation (Brereton, 2003) was performed to estimate that five latent variables were necessary to optimize the model. In the binary coding scheme for these data, the index 1 was assigned to EVOO and index 0 to adulterated oil samples. All values lower than 0.49 correspond to an adulterated oil sample, whereas those larger than 0.51 to an EVOO sample. The results between 0.45 and 0.55 were doubtful, because they were close to the threshold 0.5 (Galtier et al., 2007). The prediction results are presented in Table 3. As can be seen, values between 0.45 and 0.55 (10\% of error) have been highlighted in gray. These values correspond to the blend oil sample that had the highest percentage $(70 \%)$ of EVOO. The remaining validation samples were predicted $100 \%$ correctly.

3.1.2.2. Classification of EVOO according to RDO. In a second stage, the PLS1-DA model was used to classify EVOO samples according to geographical origin. Fifty-two EVOO samples (by duplicate, $n=104$ ) had their RDO declared on their labels, and were divided as follows: 75\% for calibration $(n=78)$ and $25 \%$ for validation $(n=26)$. In PLS1-DA, classes are calibrated one at a time, i.e. a specific regression model for each class is built, hence six models were computed for the entire system. A binary coding scheme was carried out, allocating the value 1 for a given geographical origin and 0 for the remaining ones. The decision criterion 


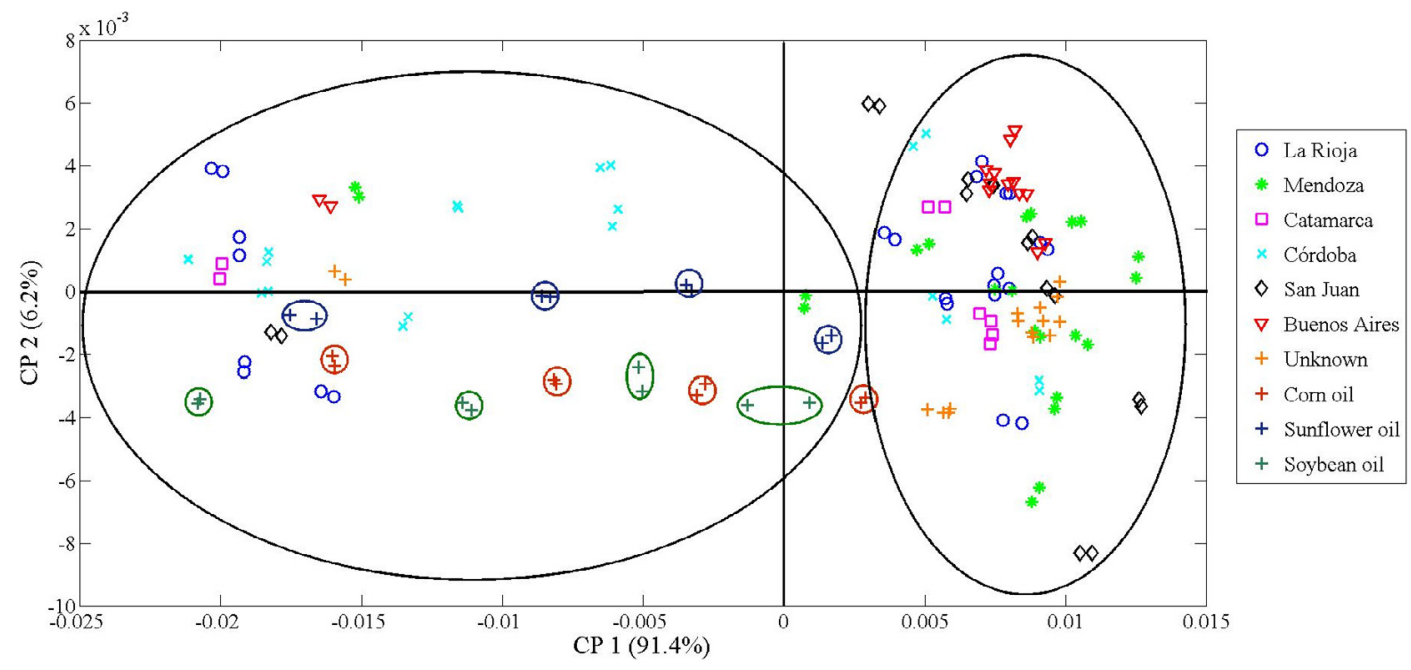

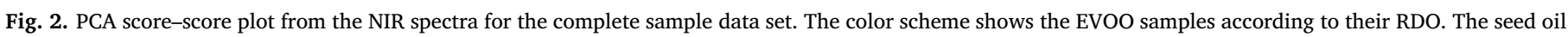
and blend samples were marked with circles: red (corn), blue (sunflower) and green (soybean).

was the same explained above in Section 3.1.2.1. The optimum number of latent variables for each origin model was estimated according to the leave-one-out cross-validation method on the calibration set (Brereton, 2003). Table 4 shows the optimum numbers of latent variables, wavelength ranges and pre-processing methods for each model, and the results obtained for the validation set using PLS1-DA analysis of the NIR spectra. As can be observed, the six origin models needed between 15 and 19 latent variables; these rather large numbers may be due to the complex chemical composition of the samples studied, and to the dispersive effects caused by the viscosity of the oil. In any case, $100 \%$ of validation samples from different RDO were well predicted by the six PLS1-DA models (highlighted in gray).

A set of eight commercial EVOO samples without any RDO declared, named as 'Unknown' in Table 1, were analyzed with the six models with the aim of evaluating their predictive abilities. Table 5 shows that the unknown samples were classified as belonging to one RDO or two RDO (i.e., blends of EVOOs from two different geographical origins) (highlighted in gray). In addition, samples 111-112 and 113-114 were of the

Table 3

Prediction results of EVOO and adulterated oil samples for the validation set using NIR spectra and PLS1-DA, and EEFMs and NPLS-DA.

\begin{tabular}{llllll}
\hline \multirow{2}{*}{ Class } & $\begin{array}{l}\text { Sample } \\
\text { number }\end{array}$ & \multicolumn{2}{l}{ NIR spectra / PLS1-DA } & \multicolumn{2}{l}{ EEFMs / NPLS-DA } \\
\cline { 2 - 7 } EVOO & $19-20$ & $\mathbf{1}$ & Pred $^{\mathbf{a}}$ & Ref & Pred $^{\text {a }}$ \\
\hline & $41-42$ & $\mathbf{1}$ & 0.959 & 1 & 1.059 \\
& $47-48$ & $\mathbf{1}$ & 0.889 & 1 & 1.012 \\
& $51-52$ & $\mathbf{1}$ & 0.986 & 1 & 0.822 \\
& $85-86$ & $\mathbf{1}$ & 0.683 & 1 & 0.714 \\
& $93-94$ & $\mathbf{1}$ & 1.046 & 1 & 1.116 \\
& $115-116$ & $\mathbf{1}$ & 1.025 & 1 & 0.969 \\
\hline Adulterated oils & $03-04$ & 0 & -0.096 & 1 & 0.817 \\
& $15-16$ & 0 & -0.055 & 0 & 0.098 \\
& $53-54$ & 0 & 0.001 & 0 & -0.015 \\
& $59-60$ & 0 & -0.212 & 0 & 0.175 \\
& $61-62$ & 0 & 0.051 & 0 & 0.504 \\
& $123-124$ & 0 & 0.035 & 0 & 0.020 \\
& $127-128$ & 0 & 0.541 & 0 & 0.022 \\
& $131-132$ & 0 & -0.027 & 0 & 0.428 \\
& $135-136$ & 0 & 0.495 & 0 & -0.299 \\
& $139-140$ & 0 & -0.011 & 0 & 0.322 \\
$143-144$ & 0 & 0.468 & 0 & -0.134 \\
& 143 & & 0 & 0.377 \\
\hline
\end{tabular}

Ref: reference; Pred: predicted

${ }^{a}$ Mean of two determinations 
Table 4

RDO prediction results for EVOO samples using NIR spectra and PLS1-DA.

\begin{tabular}{|c|c|c|c|c|c|c|c|c|c|c|c|c|c|}
\hline \multicolumn{2}{|l|}{ RDO } & \multicolumn{2}{|c|}{ La Rioja } & \multicolumn{2}{|c|}{ Mendoza } & \multicolumn{2}{|c|}{ Catamarca } & \multicolumn{2}{|c|}{ Córdoba } & \multicolumn{2}{|c|}{ San Juan } & \multicolumn{2}{|c|}{ Buenos Aires } \\
\hline \multicolumn{2}{|c|}{ Latent variables } & \multicolumn{2}{|l|}{16} & \multicolumn{2}{|l|}{16} & \multicolumn{2}{|l|}{17} & \multicolumn{2}{|l|}{15} & \multicolumn{2}{|l|}{18} & \multicolumn{2}{|l|}{19} \\
\hline \multicolumn{2}{|c|}{ Spectral range (nm) } & \multicolumn{2}{|c|}{$1100-2220$} & \multicolumn{2}{|c|}{$1350-2220$} & \multicolumn{2}{|c|}{$1100-1600$} & \multicolumn{2}{|c|}{$1350-2220$} & \multicolumn{2}{|c|}{$1100-2220$} & \multicolumn{2}{|c|}{$1100-2220$} \\
\hline \multicolumn{2}{|c|}{ Pre-processing } & \multicolumn{2}{|c|}{$\mathrm{SNV} ; \mathrm{d}[1,2,7]$} & \multicolumn{2}{|c|}{$\mathrm{SNV}$} & \multicolumn{2}{|c|}{$\mathrm{SNV} ; \mathrm{d}[1,2,7]$} & \multicolumn{2}{|c|}{$\mathrm{SNV} ; \mathrm{d}[1,2,7]$} & \multicolumn{2}{|c|}{$\mathrm{SNV} ; \mathrm{d}[1,2,7]$} & \multicolumn{2}{|c|}{$\mathrm{SNV} ; \mathrm{d}[1,2,7]$} \\
\hline RDO & $\begin{array}{l}\text { Sample } \\
\text { number }\end{array}$ & Ref & Pred $^{i}$ & Ref & Pred $^{\mathrm{a}}$ & Ref & Pred $^{\mathrm{i}}$ & Ref & Pred $^{\text {in }}$ & Ref & Pred $^{4}$ & Ref & Pred $^{\mathrm{a}}$ \\
\hline \multirow[t]{3}{*}{ La Rioja } & $1-2$ & 1 & 0.980 & 0 & -0.051 & 0 & 0.141 & 0 & -0.061 & 0 & 0.035 & 0 & 0.017 \\
\hline & $3-4$ & 1 & 0.939 & 0 & -0.018 & 0 & 0.065 & 0 & 0.042 & 0 & 0.011 & 0 & -0.027 \\
\hline & $7-8$ & 1 & 0.858 & 0 & -0.478 & 0 & 0.087 & 0 & 0.122 & 0 & -0.153 & 0 & -0.269 \\
\hline \multirow[t]{3}{*}{ Mendoza } & $37-38$ & 0 & 0.281 & 1 & 1.006 & 0 & -0.141 & 0 & -0.270 & 0 & -0.174 & 0 & -0.051 \\
\hline & $43-44$ & 0 & 0.203 & 1 & 0.949 & 0 & -0.425 & 0 & -0.319 & 0 & -0.100 & 0 & 0.031 \\
\hline & $45-46$ & 0 & 0.110 & 1 & 0.997 & 0 & 0.168 & 0 & 0.163 & 0 & -0.291 & 0 & 0.150 \\
\hline Catamarca & $53-54$ & 0 & 0.087 & 0 & -0.180 & 1 & 0.901 & 0 & 0.278 & 0 & 0.015 & $\overline{0}$ & 0.072 \\
\hline \multirow[t]{2}{*}{ Córdoba } & $65-66$ & 0 & 0.091 & 0 & 0.147 & 0 & -0.010 & 1 & 0.826 & 0 & -0.139 & 0 & -0.007 \\
\hline & $67-68$ & 0 & 0.121 & 0 & 0.226 & 0 & -0.119 & 1 & 1.032 & 0 & -0.174 & 0 & 0.141 \\
\hline \multirow[t]{2}{*}{ San Juan } & $81-82$ & 0 & 0.151 & 0 & -0.066 & 0 & 0.088 & 0 & 0.022 & 1 & 0.871 & 0 & 0.198 \\
\hline & $89-90$ & 0 & 0.133 & 0 & -0.172 & 0 & 0.114 & 0 & 0.210 & 1 & 0.912 & 0 & 0.267 \\
\hline \multirow{2}{*}{$\begin{array}{l}\text { Buenos } \\
\text { Aires }\end{array}$} & $95-96$ & 0 & -0.282 & 0 & -0.023 & 0 & -0.192 & 0 & -0.032 & 0 & 0.088 & 1 & 0.926 \\
\hline & $101-102$ & 0 & -0.530 & 0 & 0.344 & 0 & -0.124 & 0 & -0.066 & 0 & 0.054 & 1 & 0.758 \\
\hline
\end{tabular}

SNV: standard normal variate; $\mathrm{d}[1,2,7]$ : derivative [derivative order, polynomial degree, moving window]; Ref: reference; Pred: predicted

${ }^{a}$ Mean of two determinations

same trademark acquired in different markets, and in both cases the classification gave the same RDO: "La Rioja".

\subsection{Fluorescence spectroscopy}

\subsection{1. $P C A$}

Fig. 3 shows the EEFMs recorded for a typical EVOO sample, a corn oil, a sunflower oil and a soybean oil. As can be observed, only EVOO presents two bands with excitation at $368-420 \mathrm{~nm}$ and emission at 500-680 nm, correlated to vitamins and chlorophyll derivatives, respectively (Zandomeneghi et al., 2005). In contrast, the remaining seed oils present one intense band with excitation at $360 \mathrm{~nm}$ and emission at $430 \mathrm{~nm}$.

In a first phase, the PARAFAC model with non-negativity constraint in all modes was applied in the complete wavelength range to a three-way array of EEFMs measured for the studied oil samples. The number of responsive components was estimated using the consideration of the residual fit of the PARAFAC model as the number of components was increased (Arancibia et al., 2008). The progression of the residual fit values suggested 12 components. In addition to the resolved excitation and emission spectral profiles, PARAFAC renders a matrix data with the scores values for each component in each sample. The fingerprint information obtained was arranged into a matrix of size $144 \times 12$ (144 samples $\times 12$ components), which was employed with PCA for exploratory purposes.

Fig. 4 shows the score-score plot of PC1 (70.9\%) vs. PC2 (11.7\%). As can be seen there is a tendency to form two groups, one for the pure EVOOs and the second for other seed oils, blends and adulterated EVOO samples. These results are consistent with those obtained in the PCA model of NIR spectral data (Fig. 2). The color scheme in Fig. 4 is the same as that in Fig. 2. As was previously the case, the differentiation of EVOO by their RDO was not achieved.

\subsubsection{NPLS-DA}

3.2.2.1. Classification of EVOO and adulterated olive oils. The complete data set $(n=144)$ was analyzed by processing the second-order EEFM data with the NPLS-DA model. The same calibration $(n=108)$ and validation set $(n=36)$ were used, as in Section 3.1.2.1. The optimum number of latent variables was estimated according to the cross-validation method (Brereton, 2003). Calibration was performed 
Table 5

RDO prediction results of unknown EVOO samples using NIR spectra and PLS1-DA.

\begin{tabular}{lllllllllllll}
\hline RDO & \multicolumn{2}{l}{ La Rioja } & \multicolumn{2}{l}{ Mendoza } & \multicolumn{2}{l}{ Catanarca } & Córdoba & \multicolumn{2}{l}{ San Juan } & \multicolumn{2}{l}{ Buenos Aires } \\
\hline $\begin{array}{l}\text { Sample } \\
\text { number }\end{array}$ & Ref & Pred $^{2}$ & Ref & Pred $^{2}$ & Ref & Pred $^{2}$ & Ref & Pred $^{2}$ & Ref & Pred $^{2}$ & Ref & Pred $^{2}$ \\
\hline $105-106$ & 0 & 0.314 & 0 & -0.158 & 0 & 0.561 & 0 & 0.300 & 0 & 0.409 & 0 & 0.098 \\
$107-108$ & 0 & -0.263 & 0 & 1.112 & 0 & 0.312 & 0 & -0.096 & 0 & -0.107 & 0 & -0.311 \\
$109-110$ & 0 & 0.520 & 0 & 0.547 & 0 & 0.360 & 0 & 0.018 & 0 & -0.158 & 0 & -0.105 \\
$111-112$ & 0 & 0.753 & 0 & -0.088 & 0 & 0.313 & 0 & 0.134 & 0 & -0.059 & 0 & -0.045 \\
$113-114$ & 0 & 0.564 & 0 & -0.751 & 0 & 0.270 & 0 & 0.073 & 0 & 0.446 & 0 & 0.154 \\
$115-116$ & 0 & -0.209 & 0 & 0.410 & 0 & 0.373 & 0 & 0.006 & 0 & 0.609 & 0 & 0.167 \\
$117-118$ & 0 & 0.821 & 0 & 0.695 & 0 & 0.112 & 0 & -0.311 & 0 & -0.372 & 0 & -0.269 \\
$119-120$ & 0 & 0.062 & 0 & 1.030 & 0 & 0.364 & 0 & -0.043 & 0 & -0.257 & 0 & -0.523 \\
\hline
\end{tabular}

Ref: reference; Pred: predicted

${ }^{\mathrm{a}}$ Mean of two determinations

(A)

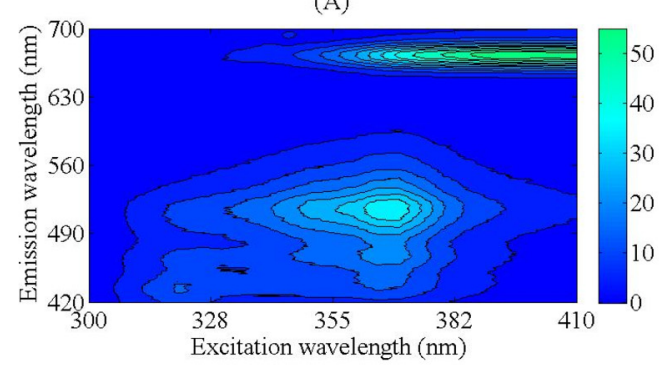

(C)

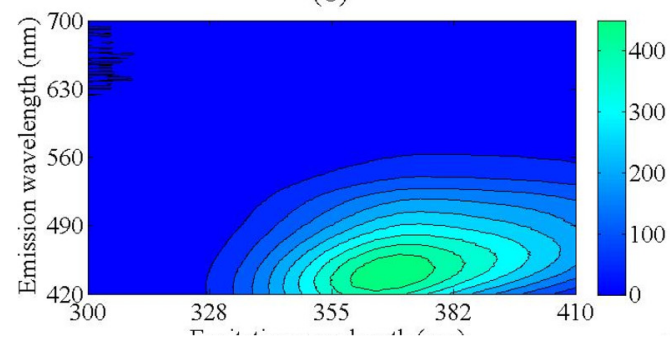

(B)

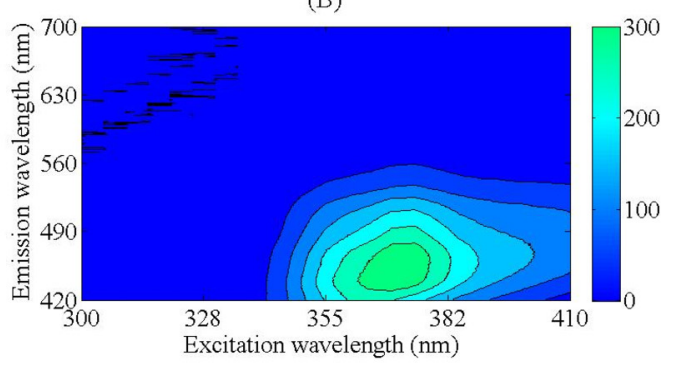

(D)

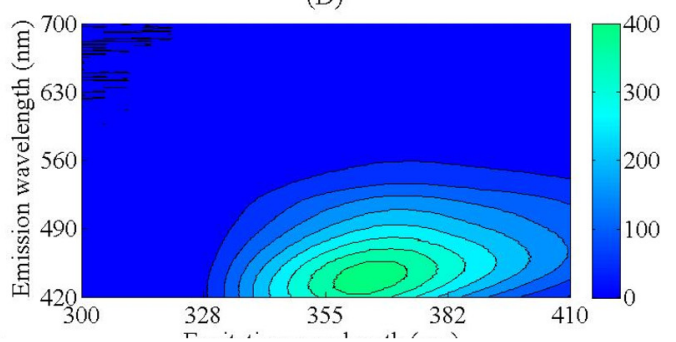

Fig. 3. Contour plots of EEFMs for (A) a randomly selected EVOO sample, (B) a corn oil sample, (C) a sunflower oil sample and (D) a soybean oil sample.

using 10 latent variables. The binary coding scheme used for these data was the same used for the NIR data, as explained above in Section 3.1.2.1. Table 3 shows the prediction results, the only validation sample that gave an incorrect value was sample 59-60 (highlighted in gray), which is within the $10 \%$ of error in prediction, while the remaining validation samples were well predicted.

3.2.2.2. Classification of EVOO according to RDO. The results obtained by applying NPLS-DA to the EEFM data with the aim of classifying EVOO according to their RDO were not satisfactory. Different spectral ranges were analyzed, but the achieved results were incorrect, and were not comparable to those furnished by with NIR spectroscopy. Literature reports indicate that EEFMs were successfully used to classify EVOO only according to their varietal or purity (Durán Merás et al., 2018; Guimet et al., 2004; Poulli et al., 2005; Zandomeneghi et al., 2005), but to the best of our knowledge they were not classified according to their origin, as is presently discussed.

\section{Conclusions}

In this report, near infrared (NIR) and fluorescence emission spectroscopies were combined with chemometric tools and applied to authenticate and validate the registered designation of origin (RDO) of Argentinean extra virgin olive oil (EVOO) samples. First-order near infrared spectral data were processed with principal component analysis (PCA) and partial least squares-discriminant analysis (PLS1-DA), while second-order fluorescence excitation-emission data were processed with PCA and the multidimensional version of PLS1-DA (NPLS-DA). After studying sixty EVOO samples, fifty-two of known and eight of unknown RDO, and artificially created adulterations with edible oils, the results showed that: (1) both NIR and fluorescence data sets were useful to detect adulterated EVOO samples from cheaper vegetable edible oils, and (2) the combination of NIR spectroscopy with PLS1-DA was the only one allowing to verify the $100 \%$ of the RDO declared in the label of each studied sample and to classify the 


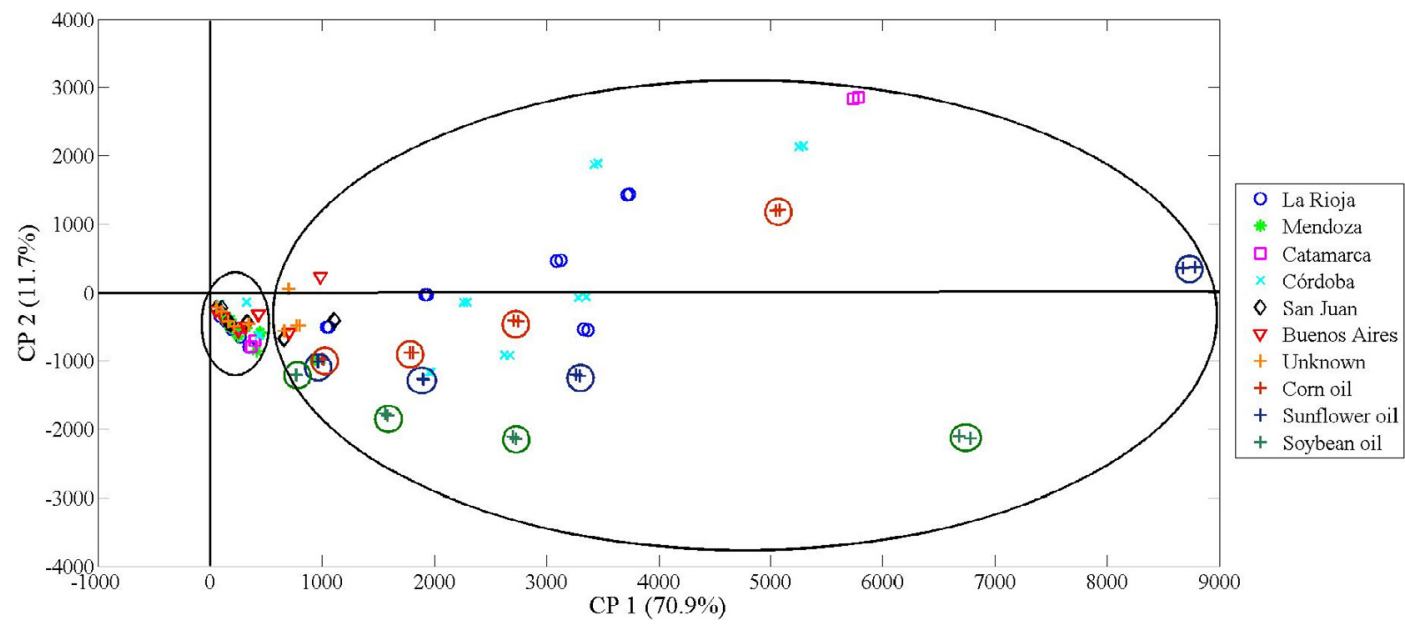

Fig. 4. PCA score-score plot from the EEFMs for the complete sample data set. The color scheme shows the EVOO samples according to their RDO. The seed oil and blend samples were marked with circles: red (corn), blue (sunflower) and green (soybean).

unknown samples as belonging to one RDO or two RDO.

\section{Acknowledgments}

The authors gratefully acknowledge the Universidad Nacional de Rosario (UNR, Project 19B/487 and BIO237), Agencia Nacional de Promoción Científica y Tecnológica (ANPCyT, Project PICT 2016-1122 and PICT 2016-1400) and Consejo Nacional de Investigaciones Científicas y Técnicas (CONICET, Project PIP 0163) for funding this work. A. M. Jiménez-Carvelo thanks Banco Santander and Universidad de Granada for a fellowship.

\section{References}

Abdi, H., \& Williams, L. J. (2010). Principal component analysis. Wiley Interdisciplinary Reviews: Computational Statistics, 2(4), 433-459.

ANMAT (2018). National Administration of Medicines, Food and Medical Technology. http:// www.anmat.gov.ar/webanmat/comunicados/ultimos_comunicados.asp/, Accessed date: 22 May 2018.

Arancibia, J. A., Boschetti, C. E., Olivieri, A. C., \& Escandar, G. M. (2008). Screening of oil samples on the basis of excitation-emission room-temperature phosphorescence data and multiway chemometric techniques. Introducing the second-order advantage in a classification study. Analytical Chemistry, 80(8), 2789-2798.

Argentinean Food Code (CAA) chapter V. (2017). http://www.anmat.gov.ar/alimentos/ normativas_alimentos_caa.asp/Accessed 22 May 2018.

Argentinean Food Code (CAA) chapter VII. (2017). http://www.anmat.gov.ar/alimentos/ normativas_alimentos_caa.asp/Accessed 22 May 2018.

ASSAI (2018). Santafe Agency for Food Security. https://www.assal.gov.ar/assal_principal/ moduloControl/noticiaSearch/index.php?tipo =1/, Accessed date: 22 May 2018.

Barker, M., \& Rayens, W. (2003). Partial least squares for discrimination. Journal of Chemometrics, 17(3), 166-173.

Brereton, R. G. (2003). In Wiley (Ed.). Chemometrics: Data analysis for the laboratory and chemical plant (Chichester, UK).

Bro, R. (1997). PARAFAC. Tutorial and applications. Chemometrics and Intelligent Laboratory Systems, 38(2), 149-171.

Bro, R., \& Smilde, A. K. (2014). Principal component analysis. Anal. Methods, 6(9), $2812-2831$.

Cercaci, L., Rodriguez-Estrada, M. T., \& Lercker, G. (2003). Solid-phase extraction-thinlayer chromatography-gas chromatography method for the detection of hazelnut oil in olive oils by determination of esterified sterols. Journal of Chromatography A, 985(1-2), 211-220.

Durán Merás, I., Domínguez Manzano, J., Airado Rodríguez, D., \& Muñoz de la Peña, A. (2018). Detection and quantification of extra virgin olive oil adulteration by means of autofluorescence excitation-emission profiles combined with multi-way classification. Talanta, 178, 751-762.

Ellis, D. I., Brewster, V. L., Dunn, W. B., Allwood, J. W., Golovanov, A. P., \& Goodacre, R.
(2012). Fingerprinting food: Current technologies for the detection of food adulteration and contamination. Chemical Society Reviews, 41(17), 5706-5727.

Fasciotti, M., \& Pereira Netto, A. D. (2010). Optimization and application of methods of triacylglycerol evaluation for characterization of olive oil adulteration by soybean oil with HPLC-APCI-MS-MS. Talanta, 81(3), 1116-1125.

Firestone, D. (2001). Assuring the integrity of olive oil products. Journal of AOAC International, 84, 176-180.

Flores, G., Ruiz Del Castillo, M. L., Blanch, G. P., \& Herraiz, M. (2006). Detection of the adulteration of olive oils by solid phase microextraction and multidimensional gas chromatography. Food Chemistry, 97(2), 336-342.

Galtier, O., Dupuy, N., Le Dréau, Y., Ollivier, D., Pinatel, C., Kister, J., et al. (2007). Geographic origins and compositions of virgin olive oils determinated by chemometric analysis of NIR spectra. Analytica Chimica Acta, 595(1-2 SPEC. ISS.), 136-144.

Gałuszka, A., Migaszewski, Z., \& Namieśnik, J. (2013). The 12 principles of green analytical chemistry and the SIGNIFICANCE mnemonic of green analytical practices. TRAC Trends in Analytical Chemistry, 50, 78-84.

Guimet, F., Ferré, J., Boqué, R., \& Rius, F. X. (2004). Application of unfold principal component analysis and parallel factor analysis to the exploratory analysis of olive oils by means of excitation-emission matrix fluorescence spectroscopy. Analytica Chimica Acta, 515(1), 75-85.

IOC (2011). The local olive grzk.owing industry in Argentina: Development, prospects and potential. Olivae, 116, 32-39. http://www.internationaloliveoil.org/store/view/ 54-olivae-116-english/, Accessed date: 22 May 2018.

Jabeur, H., Zribi, A., Makni, J., Rebai, A., Abdelhedi, R., \& Bouaziz, M. (2014). Detection of chemlali extra-virgin olive oil adulteration mixed with soybean oil, corn oil, and sunflower oil by using GC and HPLC. Journal of Agricultural and Food Chemistry, 62(21), 4893-4904.

MATLAB, 7.10. (2010). The MathWorks Inc., Natick, MA, USA.

Moros, J., Garrigues, S., \& Guardia, M. de la (2010). Vibrational spectroscopy provides a green tool for multi-component analysis. TRAC Trends in Analytical Chemistry, 29(7), 578-591.

MVC1 (2018). Program for first order multivariate calibration in MATLAB. http://www. iquir-conicet.gov.ar/esp/div5.php?area =12/, Accessed date: 22 May 2018.

Nunes, C. A. (2014). Vibrational spectroscopy and chemometrics to assess authenticity, adulteration and intrinsic quality parameters of edible oils and fats. Food Research International, 60, 255-261.

Olivieri, A. C., Goicoechea, H. C., \& Iñón, F. A. (2004). MVC1: An integrated MatLab toolbox for first-order multivariate calibration. Chemometrics and Intelligent Laboratory Systems, 73(2), 189-197.

Olivieri, A. C., Wu, H. L., \& Yu, R. Q. (2009). MVC2: A MATLAB graphical interface toolbox for second-order multivariate calibration. Chemometrics and Intelligent Laboratory Systems, 96(2), 246-251.

Poulli, K. I., Mousdis, G. A., \& Georgiou, C. A. (2005). Classification of edible and lampante virgin olive oil based on synchronous fluorescence and total luminescence spectroscopy. Analytica Chimica Acta, 542(2), 151-156.

Woodcock, T., Downey, G., \& O'Donnell, C. P. (2008). Confirmation of declared provenance of European extra virgin olive oil samples by NIR spectroscopy. Journal of Agricultural and Food Chemistry, 56(23), 11520-11525.

Zandomeneghi, M., Carbonaro, L., \& Caffarata, C. (2005). Fluorescence of vegetable oils: Olive oils. Journal of Agricultural and Food Chemistry, 53(3), 759-766. 